\title{
Das Medizinalberufegesetz (MedBG) tritt am 1.1.2018 in Kraft
}

\author{
Christoph Hänggelia ${ }^{a}$ Hanspeter Kuhn ${ }^{b}$
}

a Rechtsanwalt, Geschäftsführer des SIWF; b Fürsprecher, Abteilungsleiter Rechtsdienst FMH

Am 1.1.2018 tritt das revidierte Medizinalberufegesetz (MedBG) endgültig in Kraft. $\mathrm{Ab}$ diesem Zeitpunkt dürfen Ärztinnen und Ärzte ihren Beruf in der Schweiz nur noch dann ausüben, wenn sie im Medizinalberuferegister (MedReg) eingetragen sind. Gleichzeitig müssen alle berufstätigen Ärztinnen und Ärzte über die notwendigen Sprachkenntnisse verfügen. Für Inhaber von eidgenössischen oder von der Medizinalberufekommission (MEBEKO) formell anerkannten EU-Diplomen bzw. Weiterbildungstiteln besteht kein Handlungsbedarf. Ihre Diplome und Sprachkenntnisse werden automatisch im Register eingetragen.

Bald sind drei Jahre vergangen, seit die eidgenössischen Räte am 20. März 2015 die Revision des Medizinalberufegesetzes (MedBG) definitiv verabschiedet haben. Dank der Unterstützung durch die anderen im MedBG reglementierten Berufe ist es der FMH und dem SIWF gelungen, zwei wichtige Anliegen für den Patientenschutz zu verankern: Einerseits müssen Ärztinnen und Ärzte vor Aufnahme einer beruflichen Tätigkeit in der Schweiz im offiziellen Medizinalberuferegister (MedReg) registriert sein, und andererseits müssen sie die für die jeweilige Berufsausübung notwendigen Sprachkenntnisse nachweisen. Der Bundesrat hat die notwendigen Ausführungsverordnungen inzwischen erlassen, und das Bundesamt für Gesundheit hat die wichtigsten Informationen für die konkrete Umsetzung auf der Website publiziert.

\section{Registrierung des Arztdiploms}

Wer in der Schweiz ab dem 1.1.2018 neu eine ärztliche Tätigkeit aufnehmen will, muss sich zwingend im MedReg eintragen lassen, bevor er mit der Tätigkeit beginnt.

Wer über ein eidgenössisches Arztdiplom oder über ein von der MEBEKO formell anerkanntes EU-Arztdiplom verfügt, hat keinen Handlungsbedarf, weil diese Diplome automatisch ins MedReg eingetragen werden. Handlungsbedarf besteht für Inhaberinnen und Inhaber von

- EU-Arztdiplomen, welche sie von der MEBEKO noch nicht haben anerkennen lassen;
- nicht anerkennbaren ausländischen Arztdiplomen. Ein solches Diplom wird nach Überprüfung nur dann ins MedReg eingetragen, wenn es

- im Ausstellungsstaat zur Ausübung des Arztberufes unter fachlicher Aufsicht berechtigt und

- auf einer Ausbildung von mindestens sechs Jahren Vollzeitstudium oder 5500 Stunden theoretischen und praktischen Unterrichts an einer Universität oder einer Hochschule mit anerkanntem gleichwertigem Niveau beruht.

Wer die Bedingungen für die Registrierung nicht erfüllt erhält eine negative Verfügung. Wer das eidg. Diplom erwerben will, muss ein Gesuch an die MEBEKO stellen, welche anschliessend die Voraussetzungen für den Erwerb des eidgenössischen Arztdiploms festlegt.

Übergangsregelung: Wer mit einem ausländischen Arztdiplom bereits vor dem 1.1.2018 als Arzt in der Schweiz tätig war, hat zwei Jahre Zeit, sein Diplom von der MEBEKO anerkennen bzw. überprüfen zu lassen.

Der zuständige Kanton kann Disziplinarmassnahmen ergreifen gegen Personen, welche den Arztberuf ausüben ohne im MedReg eingetragen zu sein. Arbeitgeber sind verpflichtet, bei der Anstellung zu prüfen, ob die einzustellende Person im MedReg eingetragen ist. Wer einen nicht registrierten Arzt beschäftigt, kann mit Busse bestraft werden. 
1 Nach bisherigem MedBG musste nur der selbständig erwerbende Arzt eine Berufsausübungsbewilligung haben. In den meisten Kantonen verlangte aber schon bisher das kantonale Gesundheitsrecht, dass auch der eigenverantwortlich in einer HMO oder anderen ambulanten privaten Einrichtungen der Gesundheitsversorgung tätige Arzt über eine Berufsausübungsbewilligung verfügen musste.

2 «nach Massgabe der Art und des Umfanges der Risiken [...], die mit ihrer Tätigkeit verbunden sind».

Korrespondenz:

Christoph Hänggeli ch[at]fmh.ch

Hanspeter Kuhn lex[at]fmh.ch

\section{Registrierung der Sprachkenntnisse}

Wer den Arztberuf in der Schweiz ausübt, muss über die notwendigen Sprachkenntnisse für die jeweilige Berufsausübung verfügen. Welche Sprache und welches Niveau erforderlich sind, hängt von der konkreten Tätigkeit ab und wird vom Arbeitgeber im Einzelfall bestimmt. Das Mindestniveau ist das im gemeinsamen europäischen Referenzrahmen für Sprachen definierte Niveau B2.

Ärztinnen und Ärzte, welche privatwirtschaftlich in eigener fachlicher Verantwortung ihren Beruf ausüben, benötigen eine kantonale Berufsausübungsbewilligung (BAB). Die zuständige Gesundheitsdirektion erteilt die BAB nur dann, wenn die notwendigen Kenntnisse einer Amtssprache des Kantons nachgewiesen sind (mindestens Niveau B2).

Auf Antrag werden alle Sprachen eingetragen, für die mindestens das Niveau B2 nachgewiesen wird. Als Nachweis dienen international anerkannte Sprachdiplome (nicht älter als sechs Jahre), ein in der entsprechenden Sprache erworbener Aus- oder Weiterbildungsabschluss oder Arbeitserfahrung in der entsprechenden Sprache von drei Jahren innerhalb der letzten zehn Jahre.

Sprachkenntnisse von bereits im MedReg registrierten Ärztinnen und Ärzten werden automatisch eingetragen (entsprechend dem mit Arztdiplom bzw. Weiterbildungstitel ausgewiesenen Abschluss).

Absolventen und Absolventinnen der eidgenössischen Arztprüfung erhalten auch in Zukunft automatisch die Sprache eingetragen, in der sie die Ausbildung absolviert haben.

\section{3. Änderungen bei Berufsausübungs- bewilligung und Haftpflichtversicherung}

Neu verlangt das MedBG ab 1.1.2018, dass auch angestellte Ärztinnen und Ärzte über eine Berufsausübungsbewilligung verfügen müssen, wenn sie "privatwirtschaftlich in eigener fachlicher Verantwortung", also als Arbeitnehmer in Gruppenpraxen, HMO etc. tätig sind. ${ }^{1}$ Auch in Zukunft regelt hingegen allein das kantonale Gesundheitsrecht, für welche Funktionen im öffentlichen Spital eine Berufsausübungsbewilligung vorgeschrieben ist.

Ab 1.1.2018 fällt zudem die Möglichkeit weg, statt einer adäquaten ${ }^{2}$ Berufshaftpflichtversicherungspolice "gleichwertige andere Sicherheiten zu erbringen».

Alle weiteren Informationen finden Sie auf der Website des BAG unter www.bag.admin.ch $\rightarrow$ Themen $\rightarrow$ Berufe im Gesundheitswesen $\rightarrow$ Medizinalberufe $\rightarrow$ Revision des Medizinalberufegesetzes (MedBG).

\section{Für weiterführende Fragen}

Bundesamt für Gesundheit BAG

MEBEKO

Geschäftsstelle Ressort Ausbildung

$\mathrm{CH}-3003$ Bern

Tel. 0584629483

mebeko-ausbildung[at]bag.admin.ch 